\title{
„Normal - anders - krank“: Begriffsklärungen und theoretische Grundlagen zum Krankheitsbegriff
}

Peter Hucklenbroich

\section{Normal, anders, krank?}

Die Trias der Begriffe „,normal“, „anders“ und „krank “ beinhaltet in vollständiger Darstellung drei Paare von Unterscheidungen, nämlich zwischen normal und krank, zwischen normal und anders und zwischen anders und krank. In wissenschaftlichen Kontexten gehört nur die erste Unterscheidung im engeren Sinne zur Krankheitstheorie und damit zur Medizin; die zweite Unterscheidung gehört disziplinär eher in den Bereich einer Soziologie und Sozialpsychologie (oder, ganz abstrakt, in die Statistik) und die dritte in eine vergleichende oder abgrenzende, z. B. wissenschaftstheoretische Betrachtung zwischen den beiden ersten Unterscheidungen. Im Folgenden soll versucht werden, insbesondere die erste und die dritte Unterscheidung auf der Basis medizin- und wissenschaftstheoretischer Überlegungen näher zu charakterisieren. Eine historische oder empirisch-sozialwissenschaftliche Untersuchung der Unterscheidung zwischen Normalität und „Anderssein“ liegt dagegen nicht in der Reichweite dieses Aufsatzes; hier werde ich mich darauf beschränken, die Existenz sozialer Ab- und Ausgrenzungsphänomene als Faktum anzunehmen, ohne in tiefergehende theoretische Erklärungen und Deutungen einzusteigen.

Die folgende Untersuchung setzt sich daher aus zwei Teilen zusammen: Im ersten Teil lege ich dar, wie die Unterscheidung zwischen normal (bzw. gesund oder ungestört) und krank (bzw. krankhaft oder pathologisch) - also insbesondere der Krankheitsbegriff und der Normalitätsbegriff - in der Medizin begründet werden können. Im zweiten Teil diskutiere ich (a) einige Probleme, die sich aus dem Unterschied zwischen dem medizinischen und dem lebensweltlichen Norma- 
litätsbegriff ergeben, (b) die Frage, inwieweit historische Verschiebungen in der medizinischen Krankheitslehre als Veränderungen, Wechsel oder Relativierung des Krankheits- und Normalitätsbegriffs aufzufassen sind.

\section{Der Krankheitsbegriff}

Der Krankheitsbegriff und die auf ihm basierenden Unterscheidungen bilden die zentrale theoretische Begrifflichkeit der Medizin, vergleichbar der Rolle des Lebensbegriffs in der Biologie, des Stoffbegriffs in der Chemie oder der Begriffe von Raum, Zeit, Masse und Kraft in der klassischen Physik. Zugleich bildet der Krankheitsbegriff das wichtigste Bindeglied zwischen theoretischer Medizin und klinisch-praktischer Medizin, insofern die Krankhaftigkeit von Zuständen und Prozessen - allgemein: von Merkmalen - des menschlichen Organismus ein prima-facie-Kriterium für deren Behandlungsbedürftigkeit darstellt. (Um Missverständnisse zu vermeiden, sei betont: Krankhaftigkeit ist nicht das einzige Kriterium für Behandlungsbedürftigkeit, sondern es gibt andere Kriterien, z. B. bei normalen Altersbeschwerden. Außerdem handelt es sich um ein Kriterium prima facie, was bedeutet, dass für die Stellung einer Behandlungsindikation und die Fällung einer Behandlungsentscheidung noch weitere Kriterien zu berücksichtigen sind, so die vergleichende Bewertung der verfügbaren Behandlungsoptionen und die Präferenzen und Wertüberzeugungen von Patient und Arzt.) Daher ist die medizinische Krankheitslehre, oder genauer: die Gesamtheit von medizinischer Orthologie, Pathologie und Nosologie, das eigentliche theoretische Rückgrat der Medizin, an dem die methodischen und praktischen Anteile der Medizin wie Therapeutik und Diagnostik festgemacht sind.

Während nun die medizinische Fachliteratur einschließlich der üblichen Lehr- und Handbücher das Erfahrungsmaterial bezüglich der einzelnen gesunden und krankhaften Lebenserscheinungen am menschlichen Organismus ausgiebig behandelt, fehlt eine entsprechende Darstellung der dem ganzen System zugrunde liegenden Unterscheidung zwischen gesund (bzw. normal) und krank (bzw. krankhaft). Es existieren umfangreiche Darstellungen z. B. der normalen und Pathologischen Anatomie, der Physiologie und Pathophysiologie oder der klinischen Subdisziplinen wie Innere Medizin, Chirurgie und Psychiatrie; dagegen gibt es kein Lehrbuch und keine Standarddarstellung der Theoretischen Pathologie - wenn man mit Karl E. Rothschuh und der Heidelberger Schule um Wilhelm Doerr und Heinrich Schipperges die für den allgemeinen Krankheitsbegriff benötigte und zuständige Disziplin so bezeichnen will. ${ }^{1}$ Stattdessen finden wir allerdings ausgedehnte Diskussionen darüber, wie ein allgemeiner Krankheitsbegriff theoretisch zu fassen wäre. Diese Diskussionen haben zwar schon eine lange medizinhistorische Tradition bis zurück zum Corpus Hippocraticum, ${ }^{2}$ haben aber etwa seit den 1970er Jahren eine neue Qualität gewonnen: Es beteiligen sich nunmehr zunehmend nicht nur Mediziner, sondern auch Vertreter anderer Fächer wie Philosophie, Psychologie, Soziologie, (Medizin-)Geschichte und (Bio-)Ethik, und die Diskussion wird

1 Rothschuh (1958); Doerr/Schipperges (1979); Becker/Doerr/Schipperges (1993).

2 Vgl. die in den Sammelbänden Rothschuh (1975b) und Caplan/Engelhardt/McCartney (1981) zusammengestellten historischen Zeugnisse zu dieser Diskussion. 
international geführt. Man kann sogar den Eindruck gewinnen, dass sich diese neuere Diskussion bislang eher außerhalb als innerhalb der Medizinischen Fakultäten abgespielt hat, insofern als Autoren wie Christopher Boorse, Bernard Gert, Lawrie Reznek oder Lennart Nordenfelt in der Philosophie und den Sozialwissenschaften bekannter zu sein scheinen als in der Medizin. Es handelt sich aber um eine Diskussion, die die begrifflichen und theoretischen Grundlagen der Medizin direkt betrifft, gewissermaßen um die medizintheoretische Grundlagenforschung - oder den medizinischen Grundlagenstreit. ${ }^{3}$

Während eine Zeit lang der Eindruck entstehen konnte, dass diese Grundlagendiskussion aporetisch und extrem kontrovers verläuft, lassen sich mittlerweile doch bestimmte Resultate festhalten, die nur mehr wenig kontrovers sind und in die Richtung einer vereinheitlichten allgemeinen Krankheitstheorie mit einem in bestimmter Weise fassbaren allgemeinen Krankheitsbegriff weisen. Ohne behaupten zu wollen, dass sich bereits ein allgemeiner Konsens in dieser Hinsicht abzeichne, glaube ich doch die Grundlinien dieser Theorie angeben zu können, insoweit sie aus einer kritischen Rekonstruktion der Struktur der medizinischen Krankheitslehre selbst abgeleitet werden können. Ich lege diese Konzeption der Theoretischen Pathologie, die an anderer Stelle ausführlich entwickelt wurde, ${ }^{4}$ der folgenden kurzgefassten Darstellung zugrunde.

\subsection{Grundlagen}

Wie schon Karl E. Rothschuh aufgezeigt hat, ${ }^{5}$ gibt es nicht nur den Krankheitsbegriff der Medizin, sondern man muss zusätzlich in Rechnung stellen, dass die Bezeichnungen krank und Krankheit

a) auch in der alltäglichen Lebenswelt gebraucht werden und dann ein Krankheitsverständnis widerspiegeln, das sich nicht mit dem der (wissenschaftlichen) Medizin deckt;

b) in der Arbeitswelt, im Sozialrecht und im Versicherungswesen einen bestimmten sozialen Status bezeichnen, der mit bestimmten Erwartungen, Rechten, Pflichten, Ansprüchen und Privilegien verbunden ist; ${ }^{6}$

c) sowohl in der Geschichte der abendländischen Medizin als auch in außereuropäischen Kulturen und Medizinsystemen der Vergangenheit und Gegenwart mit anderen theoretischen Vorstellungen und praktischen Konsequenzen verbunden wurden bzw. werden, als es in der zeitgenössischen „westlichen“ wissenschaftlichen Medizin der Fall ist. ${ }^{7}$

Es ist sehr wahrscheinlich nicht möglich, einen Bedeutungskern von krank und Krankheit zu identifizieren, der allen genannten Verwendungsweisen dieser Bezeichnungen im Sinne eines gemeinsamen Definiens zugrunde liegt. Wenn man bedenkt, dass in bestimmten Medizinsystemen z. B. persönliches Missgeschick,

3 Im Rahmen dieses Aufsatzes kann ich nicht näher auf diese ausgedehnte Diskussion eingehen. Es sei auf die im Literaturverzeichnis angeführten Werke der im Text genannten Autoren verwiesen sowie auf Caplan/Engelhardt/ McCartney (1981), Humber/Almeder (1997) und Caplan (2004).

4 Vgl. Hucklenbroich (2006), (2007a), (2007 b); Buyx/Hucklenbroich (2007); Hucklenbroich et al. (2007).

5 Rothschuh (1975a).

6 Die klassische Darstellung dazu ist Parsons (1967). Vgl. auch Mazal (1992), (2004).

7 Vgl. Schipperges/Seidler/Unschuld (1978); Lux (2003). 
Streitigkeiten mit anderen Menschen oder das Vertreten bestimmter religiöser Auffassungen zu den Krankheiten gerechnet werden, in anderen Systemen dagegen Ereignisse wie ein Schlaganfall, eine Epilepsie oder das Versterben eines Kindes nicht als Krankheiten, sondern z. B. als göttliche Strafe oder göttliche Erleuchtung aufgefasst werden, scheint eine gemeinsame begriffliche Basis nicht denkbar zu sein. Wenn man aber deswegen als „kleinsten gemeinsamen Nenner“ nur eine negative Normativität annehmen will, also die Vorstellung, dass etwas nicht so ist oder sich verhält, wie es sollte, ${ }^{8}$ erhält man wieder eine viel zu weite Bedeutung, da davon nicht nur Krankheitserscheinungen, sondern alle Arten von Unglück, Misserfolg, Unfähigkeit, Kriminalität oder Unmoral abgedeckt werden.

Aus diesem Grunde ist es sinnvoll, sich bei der Analyse des Krankheitsbegriffs bewusst auf den Bereich zu beschränken, in dem eine systematische theoretische Grundlage vorgefunden werden kann: Die wissenschaftlich-medizinische Krankheitslehre im o. g. Verständnis. Diesen medizinischen Krankheitsbegriff gilt es sodann klar zu unterscheiden vom alltagssprachlich-lebensweltlichen Begriffsverständnis, vom sozialen bzw. soziologischen Krankheitsbegriff und von den Krankheitsvorstellungen anderer Kulturen und Epochen. Der Vorteil, den man sich mit dieser selbstauferlegten Beschränkung einhandelt, besteht in der Möglichkeit, die Bedeutung dieses Krankheitsbegriffs in Form einer relativ überschaubaren und logisch klaren Definition angeben zu können. Diese Definition stellt natürlich keine normative Vorgabe an die Medizin dar, so als ob die Medizintheorie den Ärzten vorschreiben wollte, was sie unter Krankheit zu verstehen haben, sondern sie ist eine Zusammenfassung der grundlegenden Kriterien und Prinzipien, die sich in der heutigen medizinischen Krankheitslehre, als Resultat ihrer bisherigen historischen Entwicklung, finden .

Welche sind nun diese grundlegenden Kriterien und Prinzipien, und wie kann man sie erkennen? Die erste, negative Antwort hierauf besagt, dass keine der gängigen „Definitionen“ von Krankheit oder Gesundheit, wie man sie etwa in Wörterbüchern und Konversationslexika findet, eine gehaltvolle, theoretisch oder praktisch brauchbare Charakterisierung dieser Begriffe beinhaltet: Diese Worterklärungen sind entweder zirkulär („Krankheit als Abwesenheit von Gesundheit“ und „Gesundheit als Abwesenheit von Krankheit“), oder sie führen zum Regress, indem sie Begriffe zur Definition heranziehen, die selbst genau so erklärungsbedürftig sind wie das Definiendum (z. B. Krankheit als Störung, als Regelwidrigkeit, als Normabweichung oder Abnormalität), oder sie sind genau besehen inhaltsleer (z. B. Krankheit als „Leben unter veränderten Bedingungen“). Auch die etwas elaborierteren Versionen, in denen etwa Krankheit mit einer Disharmonie, Abweichung vom Gleichgewicht oder Störung der Regulations- und Kompensationsfähigkeit des Organismus gleichgesetzt wird, ${ }^{9}$ leiden unter ähnlichen Defekten: Erstens wären sie nur anwendbar, wenn unabhängig von ihnen schon klar wäre, wann denn eine ungestörte Harmonie, ein ungestörtes Gleichgewicht oder eine ungestörte Regulationsfähigkeit vorliegt; und zweitens wären sie selbst dann, wenn dies

8 Dies wurde von Axel Bauer vorgeschlagen; Bauer (2007), S. 94.

9 Definitionsvorschläge, die auf diese und ähnliche Vorstellungen zurückgreifen, finden sich z. B. in aktuellen Lehrbüchern der Pathologie wie Grundmann et al. (2004), Böcker (2004), Riede (2004). 
Wissen vorausgesetzt werden könnte, keineswegs allgemeingültig, sondern würden zwar auf einige ganz bestimmte Krankheitsklassen passen, viele andere Klassen von Krankheiten dagegen völlig unberücksichtigt lassen.

Die von mir zugrunde gelegte medizintheoretische Hypothese, auf deren Basis eine positive Antwort gegeben werden kann, lautet folgendermaßen: Um den Krankheitsbegriff der Medizin zu rekonstruieren, müssen diejenigen Voraussetzungen identifiziert werden, die in der medizinischen Beurteilung und Theoriebildung regelmäßig in Anspruch genommen werden, aber (in der Regel) implizit bleiben. Dass diese impliziten Voraussetzungen tatsächlich angenommen werden und logisch auch notwendig sind, lässt sich zeigen, sobald sie explizit formuliert sind, denn dann kann man prüfen und nachweisen, dass die geltende Krankheitslehre und Diagnostik von diesen Voraussetzungen Gebrauch macht. Es handelt sich im Wesentlichen um drei Voraussetzungen, die $z$. T. in sich noch einmal differenziert sind. Die erste Voraussetzung besteht in der Zugrundelegung eines (primären) Kriteriums für Krankhaftigkeit, das sich aus drei (bzw. fünf, s.u.) Teilkriterien zusammensetzt. Die zweite Voraussetzung besteht in dem Prinzip, dass es möglich ist, bestimmte Phänomene als Teil-, Begleit- und Folgeerscheinungen krankhafter Vorgänge zu identifizieren und daher als krankhaft zu klassifizieren, auch wenn diese Phänomene für sich allein betrachtet nicht von den primären Krankheitskriterien erfasst werden sollten: Nämlich erstens dann, wenn diese Phänomene notwendige Glieder in einer spezifischen pathogenetischen Entwicklung sind, die zu einer bereits als pathologisch bekannten Erscheinung gehört, und wenn diese Phänomene nicht außerhalb solcher pathogenetischer Entwicklungen auftreten; zweitens auch dann, wenn sie nach klinischer Erfahrung spezifischer Teilvorgang oder Symptom eines bereits als solchen identifizierten Krankheitsbildes (einer nosologischen Krankheitsentität) sind. Ergänzend tritt als dritte Voraussetzung noch das Prinzip auf, dass sich alle krankhaften Erscheinungen im Organismus prinzipiell innerhalb des Systems aller Krankheiten - der Nosologie zuordnen lassen (müssen), und zwar als Erscheinungen einer oder mehrerer vorliegender Krankheitsentitäten. Dies letzte Prinzip sichert die Vollständigkeit des medizinischen Krankheitsbegriffs und liegt insbesondere der Methode der Diagnostik und Differentialdiagnostik konstitutiv zugrunde, indem es postuliert, dass es keine „isolierten“, aus dem System der Krankheitsentitäten herausfallenden Krankheitserscheinungen gibt, sondern jedes pathologische Phänomen im Rahmen einer oder mehrerer kombinierter Krankheitsentität(en) auftritt und dementsprechend diagnostisch zugeordnet werden muss.

Man kann die gerade gegebene Rekonstruktion in Form einer Definition zusammenfassen:

(D 1) Ein Vorgang (Zustand/Ereignis) ist krankhaft (pathologisch) genau dann,

(1) wenn mindestens eines der fünf Krankheitskriterien (siehe D2) auf ihn zutrifft, oder

(2) wenn er, entsprechend dem ätiopathogenetischen Krankheitsmodell, ausgehend von ersten Krankheitsursachen ein spezifisch krankheitsbedingender Vorgang im Rahmen eines pathogenetischen Prozesses ist, oder 
(3) wenn er als Ganzes einer (oder mehreren kombinierten) nosologischen Krankheitsentität(en) entspricht oder als Teil oder Symptom unter eine (oder mehrere kombinierte) Krankheitsentität(en) subsumierbar ist.

Um diese Definition vollständig zu machen, müssen noch die primären Krankheitskriterien angegeben werden. Es handelt sich dabei um Kriterien, die sich schon in der antiken Medizin (implizit) nachweisen lassen ${ }^{10}$ und deren Grundgedanke ganz kurz und einfach formuliert werden kann: Ein Zustand oder Vorgang im Organismus ist krankhaft, wenn er (unbehandelt)

1. zum Tode führt,

2. mit Schmerz oder Leiden verbunden ist,

3. oder das (individuelle) Risiko erhöht, dass ein Ereignis der ersten oder zweiten Art auftritt.

Wenn man diese einfachen Crundgedanken so ausformuliert, dass alle möglichen - gutgläubigen oder böswilligen - Missdeutungen und Missverständnisse ausgeschlossen sind, wird die Formulierung der Definition deutlich umfangreicher und unhandlicher. Es muss insbesondere zusätzlich festgehalten werden, dass die Beurteilung eines Vorgangs als pathologisch immer einen Vergleich einschließen muss mit der Situation, die bestehen würde, wenn der fragliche Vorgang ceteris paribus nicht eingetreten wäre; und dass überhaupt die Möglichkeit eines solchen alternativen Verlaufs (nach bestem ärztlichen bzw. medizinischen Wissen) gegeben sein muss. Wenn es zu einem Vorgang gar keine natürliche Alternative gibt, oder wenn die Alternativen gemäß Kriterium (1) bis (3) ungünstiger wären als der fragliche Vorgang, dann betrachten wir einen solchen Vorgang nicht als krankhaft. Dagegen betrachten wir Vorgänge nicht schon deswegen als krankhaft, wenn es zwar bessere Alternativverläufe gibt, diese aber ausschließlich durch gezieltes menschliches (z. B. technisches oder medizinisches) Eingreifen möglich gemacht werden können (das wäre im übrigen auch nicht ceteris paribus).

Zur Erläuterung dieser abstrakten Prinzipien einige kurze Beispiele:

- Das Auftreten einer kurzfristigen Bewusstlosigkeit, z. B. als orthostatischer Kollaps bei arterieller Hypotonie oder vagovasale Synkope, ist pathologisch, weil es erstens mit Verletzungsgefahr und (kurz dauernder) Hilflosigkeit verbunden ist, die bei erhaltenem Bewusstsein nicht auftreten würden, und insofern unter Kriterium 3 fällt; und weil zweitens ohne Widerspruch zur Erfahrung angenommen werden kann, dass ein gesundes Leben ohne solche Bewusstlosigkeiten möglich ist (= dass ein Alternativverlauf möglich ist und weniger nachteilig wäre).

- Das regelmäßige Auftreten mehrstündiger Bewusstlosigkeiten jede Nacht (sog. natürlicher Schlaf) ist nicht pathologisch - obwohl es mit Hilfund Wehrlosigkeit verbunden ist -, weil erfahrungsgemäß (und wissen-

10 Eine Lektüre z.B. der hippokratischen Schrift „Die Natur des Menschen“ zeigt, dass sich hinter den expliziten humoralpathologischen Vorstellungen implizit auch schon dort mindestens die ersten beiden Krankheitskriterien finden, vgl. Hippokrates (1975). 
schaftlich erklärbar) ein Überleben ohne solchen regelmäßigen Schlaf nicht möglich ist (Alternative $=$ Tod durch Schlafentzug).

- Falls es in Zukunft möglich werden sollte, durch z. B. neuropharmakologische Maßnahmen zu erreichen, dass Menschen teilweise oder ganz ohne Schlaf auskommen, ohne gesundheitlichen Schaden zu nehmen, würde dadurch der natürliche Schlaf nicht zu einem pathologischen Phänomen, sondern - allenfalls - zu einer mehr oder weniger entbehrlichen oder obsoleten Lebensform.

Dies sind bewusst einfach gehaltene Beispiele, die trivial erscheinen mögen. Sie sollen jedoch deutlich machen, wie man auch in sehr viel komplizierteren Zusammenhängen grundsätzlich argumentieren würde.

$\mathrm{Zu}$ den drei genannten Grundkriterien treten zwei weitere Kriterien hin$\mathrm{zu}$, die sich spezifisch auf die Fähigkeit zur biologischen Reproduktion und zur Beteiligung am sozialen Leben beziehen: Wenn eine dieser Fähigkeiten eingeschränkt oder verloren ist, liegt nach den Teilkriterien 4 und 5 ebenfalls ein pathologischer Zustand oder Vorgang vor. Diese beiden Kriterien werden zwar, wie anderen Ortes gezeigt, ${ }^{11}$ in der medizinischen Krankheitslehre de facto verwendet und finden sich implizit z. B. auch in den Systematiken des ICD-10 und DSM-IV, sie sind jedoch in der interdisziplinären Diskussion um den Krankheitsbegriff deutlich kontroverser als die ersten drei Kriterien. Gerade deshalb ist es sinnvoll, sie als eigene Kriterien zu formulieren und nicht z. B. in das Kriterium 3 zu integrieren, da so die Diskussion um Sinn und Angemessenheit dieser letzten beiden Kriterien separat und transparent geführt werden kann, ohne die unkontroverseren Anteile der Rekonstruktion in Frage stellen zu müssen.

Nimmt man das bis hierhin Gesagte zusammen und versucht eine vollständige Formulierung der Krankheitskriterien zu geben, so erhält man in etwa folgende Definition:

(D 2) Ein Vorgang (Zustand/Ereignis) ist krankhaft (pathologisch) genau dann,

(1) wenn er bei natürlichem, unbehandeltem Verlauf unmittelbar zum vorzeitigen Tod oder zur Verkürzung der natürlichen Lebenserwartung des Betroffenen führt, oder

(2) wenn er (unbehandelt) mit Schmerz, Leiden, Missempfindungen oder Beschwerden in körperlicher und/oder seelischer Hinsicht verbunden ist, wobei diese Zustände bestimmte natürlich vorgegebene, kulturell überformbare Normalbereiche oder Schwellenwerte bezüglich Intensität, Dauer und/oder Häufigkeit des Auftretens überschreiten, oder

(3) wenn er das individuelle Risiko erhöht, dass ein Ereignis eintritt, das schon nach mindestens einem Krankheitskriterium (1-5) als krankhaft erkannt ist. Insbesondere: wenn er die natürlich vorgegebenen körperlich-seelischen Dispositionen und Potentiale des Betroffenen so verän$\operatorname{dert}(z$. B. Fähigkeiten einschränkt oder verschwinden lässt, ungünstige Reaktionsweisen entstehen lässt oder verstärkt), dass dieser (ohne ad-

11 Vgl. Hucklenbroich (2006), S. $18 \mathrm{ff}$. 
äquate Behandlung bzw. Substitution) in bestimmten, zuvor harmlosen Situationen krank wird (gemäß den Krankheitskriterien 1-5), z. B. in Lebensgefahr gemäß Klausel (1) gerät oder Schmerz, Leiden oder Beschwerden gemäß Klausel (2) ertragen muss, oder wenn bestimmte solche Dispositionen/Potentiale von vornherein (d. h. in angeborener Weise) in dem genannten Sinn vorhanden, verändert bzw. eingeschränkt/ verstärkt sind oder ganz fehlen, oder/und

(4) wenn er unbehandelt die Unfähigkeit zur biologischen Reproduktion beinhaltet oder zur Folge hat, oder/und

(5) wenn (a) ein einzelner davon Betroffener unbehandelt nicht in der Lage ist, mit „gesunden“ menschlichen Lebensgemeinschaften seinem Alter entsprechend in möglichst konfliktfreier und kooperativer Weise und ohne selbst Leid zu verursachen zusammenzuleben, oder (b) umgekehrt eine davon betroffene Lebensgemeinschaft „unbehandelt" nicht in der Lage ist, ihren einzelnen Mitgliedern ein möglichst leid- und konfliktfreies, kooperatives Zusammenleben zu gewährleisten.

Zusatzbedingungen: Alle fünf Kriterien gelten nur für Vorgänge, die

a) Merkmale des Organismus selbst und nicht seiner Umgebung sind,

b) keine bewussten Erkenntnisse und Absichten und keine gewollten, intentionalen Handlungen sind, sofern Wahrnehmungs- und Erkenntnisfähigkeit, Wille und Handlungsfähigkeit nicht selbst erkrankt sind,

und unter der empirisch zu belegenden Voraussetzung, dass

c) es mindestens einen natürlich vorkommenden, bei dem Betroffenen grundsätzlich möglichen alternativen Lebensprozess bzw. alternativen Verlauf gibt, bei dem der fragliche Prozess und seine Konsequenz (Tod, Leiden, ...) nicht auftritt. Der Alternativverlauf darf natürlich seinerseits kein pathologischer Verlauf sein;

d) alternative Verläufe, die nur durch gezieltes, intentionales menschliches Handeln zustande kommen können, nicht in diesen Vergleich einbezogen werden (außer bei Erkrankungen von Erkenntnis- und Selbstbestimmungsfähigkeit).

Mit den beiden Definitionen D1 und D2 zusammengenommen kann die der gesamten medizinischen Krankheitslehre zugrunde liegende Unterscheidung zwischen gesund/normal und krank/krankhaft in jedem Einzelfall nachgezeichnet und in diesem Sinne begründet werden. Das ist jedenfalls die hier vertretene These, die sich in Konfrontation mit der tatsächlichen medizinischen Lehre prüfen und bestätigen lassen muss. An dieser Stelle kann natürlich kein umfangreicher Nachweis erfolgen, sondern ich muss mich auf einige Beispiele beschränken. ${ }^{12}$

12 Ausführliche Erläuterungen zu dieser Definition habe ich in Hucklenbroich (2006) und (2007a) gegeben. 


\subsection{Allgemeine Beispiele}

Zunächst seien zur Illustration des bis hierhin Gesagten einige Krankheitsbilder angeführt, die verdeutlichen können, wie die Definitionen und die Krankheitskriterien zu verstehen sind.

Typische Beispiele für Erkrankungen, bei denen primär oder ausschließlich das Kriterium (1) zugrunde liegt, sind folgende:

1. Verletzungen mit Eröffnung großer Blutgefäße, Zerstörung lebenswichtiger Organe, Unterbindung der Blutversorgung des Gehirns

2. Vergiftungen mit starken Toxinen (Cyanide, Arsenide, Knollenblätterpilz, CO-Gas)

3. Malignome, bestimmte bakterielle und virale Infektionen (Tbc, Tollwut, Cholera, HIV)

4. Bestimmte erbliche Krankheiten wie Zystische Fibrose, Chorea Huntington, Zystennieren, letal endende Muskeldystrophien, Progeria infantum.

Typische Beispiele für Erkrankungen, bei denen primär oder ausschließlich das Kriterium (2) zugrunde liegt, sind folgende:

1. Steinerkrankungen (Nierenbecken, Gallenblase) mit Koliken

2. Weichteilrheumatismus

3. Pruritus bei Erkrankungen der Haut

4. Schmerzen und Parästhesien bei Neuritiden und Polyneuropathien

5. Depressionen

6. Phobien und Panikerkrankungen.

Typische Beispiele für Erkrankungen, bei denen primär oder ausschließlich das Kriterium (3) zugrunde liegt, sind folgende:

1. Asymptomatische Gefäßstenosen z. B. durch Arteriosklerose (KHK, AVK, Karotisstenose)

2. Herzinsuffizienz Stadium NYHA I-III

3. Myasthenien, nicht-letale Muskeldystrophien, Paresen

4. Blindheit, Farbenblindheit

5. Einnierigkeit.

Es ist jedoch darauf hinzuweisen, dass bei den meisten Erkrankungen (im Sinne von Krankheitsentitäten), die die Medizin kennt, mehr als ein Krankheitskriterium zugrunde liegt, d.h. im Verlauf einer Erkrankung treten verschiedene klinische und pathologisch-pathophysiologische Manifestationen auf, die unter unterschiedliche Krankheitskriterien fallen. Es treten also z. B. sowohl Beschwerden als auch Behinderungen (z. B. Schmerzen und Claudicatio intermittens bei peripherer AVK), oder sowohl Lebensgefährdung als auch Schmerzen (z. B. bei akuter Appendizitis) gleichzeitig auf. Nicht selten sind alle drei Kriterien gleichzeitig oder nacheinander erfüllt, also Lebensbedrohlichkeit, Schmerz/Beschwerden und Behinderungen (überlebter cerebraler Insult oder Myokardinfarkt, fortgeschrittene Malignome, schwere Unfälle). 
Beispiele für krankhafte Zustände, die für sich allein betrachtet nicht unter die Krankheitskriterien fallen, sondern erst unter Berücksichtigung ätiopathogenetischer oder klinisch-nosologischer Zusammenhänge als krankhaft erkannt werden, sind z. B. Form- und Farbveränderungen von inneren Organen oder äußeren Hautarealen, Änderungen in der Struktur von Biomolekülen oder in der Zusammensetzung von Zellen und Körperflüssigkeiten: Eine Gelbfärbung der Haut und Schleimhäute, oder eine Gelbfärbung des inneren Organs Leber, sind nicht im Sinne der Kriterien 1-5 krankhaft, aber unter Kenntnis der Zusammenhänge zwischen bestimmten Leber- und Bluterkrankungen und solchen Farbveränderungen können sie trotzdem als eindeutig pathologisch klassifiziert werden (Gelbsucht bzw. Ikterus als Symptom; Gelbfärbung der Leber als Zeichen der Fettleber). Vorauszusetzen ist bei solchen Klassifikationen natürlich, dass die zur Begründung herangezogenen Krankheiten (Krankheitsentitäten) schon unabhängig von den betrachteten Symptomen bzw. Zuständen als krankhaft erwiesen werden können, im Beispiel etwa aufgrund der Schäden und Risiken, die mit einer eingeschränkten Leberfunktion oder einem Zerfall von roten Blutzellen einhergehen.

\subsection{Beispiele und Erläuterungen zu Kriterium 4}

Zu den Zuständen, die als „Störungen der Fortpflanzungsfähigkeit“ eingeordnet werden, rechnet man insbesondere:

1. Sterilität/Infertilität („Impotentia generandi“)

2. Impotentia coeundi

3. (ungewollte) Kinderlosigkeit.

Es handelt sich also darum, dass entweder durch somatische Veränderungen oder Varianten am reproduktiven System von Mann oder Frau (Anatomie und Entwicklung der Keimdrüsen und Genitalorgane, Produktion und Proliferation von Ei- und Samenzellen, Produktion und Regulation der Geschlechtshormone, etc.) oder durch die somatisch oder psychisch zu erklärende Unfähigkeit zur Kohabitation oder aufgrund unbekannter Ursachen bei bestehendem Kinderwunsch und trotz bestehender somatischer und psychischer Voraussetzungen, es bei einer Einzelperson oder einem Paar nicht zu einer Fortpflanzung kommt bzw. kommen kann. Es kann sich also beispielsweise um die genetisch bedingte Infertilität bei Formen der Intersexualität, um eine erworbene Sterilität aufgrund von Infektionen oder Malignomen der Genitalorgane oder um eine psychosomatisch zu erklärende Unfähigkeit zur Ausübung des Geschlechtsaktes (erektile Dysfunktion oder Vaginismus) handeln.

Auch die absichtlich herbeigeführte Unfähigkeit zur Fortpflanzung, z. B. durch Vasektomie im Rahmen der Familienplanung beim Mann, führt zu einem Zustand, der grundsätzlich als krankhafte Veränderung („Verstümmelung“) einzuordnen ist. Dies kann nur dann irritieren, wenn man in einer solchen Einordnung einen Makel oder eine Abwertung des betroffenen Individuums sehen will. Die theoretisch-pathologische Klassifikation impliziert jedoch keinerlei Wertung dieser Art, sondern dient lediglich zur deskriptiven 
Unterscheidung und Ordnung der Erscheinungen. Wer damit eine (Ab-)Wertung verbindet, tut dies auf eigene Rechnung (vgl. Kapitel 4.1).

Es ist vermutet worden, dass aufgrund von Kriterium (4) bestimmte psychosexuelle Orientierungen, insbesondere die Homosexualität, als pathologisch eingeordnet werden müssten, da bei homosexuell Orientierten das Interesse an oder sogar die Fähigkeit zur heterosexuellen Betätigung vermindert oder ganz fehlend sei. Hier muss sorgfältig differenziert werden: Soweit es sich nur darum handelt, dass die sexuelle Betätigung und das sexuelle Interesse des Betreffenden sich nicht auf das andere Geschlecht richten, kann hier ebenso wenig eine Krankhaftigkeit angenommen werden wie etwa bei der Ausübung einer (Risiko-)Sportart, bei der das Risiko einer körperlichen Verletzung oder sogar des tödlichen Unfalls in relevanter Weise erhöht ist: Die Ausübung einer Sportart ist, als intentional herbeigeführte Handlung bzw. als Teil des Lebensstils, kein Kandidat für Krankheit oder Krankhaftigkeit; die Ausübung von Sexualität auch nicht. Sofern dagegen eine psychisch-psychosomatische Impotentia coeundi (in Bezug auf das andere Geschlecht) vorliegt, wäre dies zwar tatsächlich eine pathologische Kondition, aber damit wird nicht die psychosexuelle Orientierung bzw. der Lebensstil als solche(r) ebenfalls pathologisch. Richtig ist hier lediglich, dass ein Lebensstil mehr oder weniger riskant sein kann. Es lässt sich übrigens ohne weiteres denken, dass eine solche Impotentia bei entsprechendem Wunsch therapierbar ist, ohne dass die psychosexuelle Orientierung sich dabei ändert. Eine Krankhaftigkeit im Zusammenhang mit Homosexualität kann allenfalls dann angenommen werden, wenn der oder die Betreffende unter seiner/ihrer Veranlagung subjektiv leidet; dann aber ist nicht Kriterium (4), sondern Kriterium (2) einschlägig. Das heißt, dieses Leiden kann Krankheitswert besitzen; aber damit darf nicht gleichzeitig automatisch die Ursache bzw. der Anlass dieses Leidens, nämlich die Wahrnehmung eines bestimmten Zustandes oder dieser Zustand selbst, als krankhaft eingestuft werden. Dies wäre erst dann der Fall, wenn die psychosexuelle Veranlagung selbst als ein Zwang im Sinne einer Zwangserkrankung erlebt würde. In diesem Zusammenhang ist weiterhin $\mathrm{zu}$ beachten, dass Leiden unter einem bestimmten wahrgenommenen Zustand nicht verwechselt werden darf mit dem Leiden unter der gesellschaftlichen Reaktion aufdiesen Zustand; letzteres ist zwar auch ein Leiden und kann Krankheitswert besitzen, hat aber eine andere Ursache und könnte ggf. zusätzlich das Kriterium (5b) erforderlich machen (vgl. Kapitel 4.2).

\subsection{Beispiele und Erläuterungen zu Kriterium 5}

Die Krankheitskriterien (1) bis (4) decken nicht nur die somatischen, sondern auch einen großen Teil der psychischen Erkrankungen ab. So trifft das Kriterium (2) etwa für solche Störungen zu, die mit übermäßigen, inadäquaten Gefühlen von Trauer (z. B. bei Depressionen) oder Angst (z. B. bei Phobien und Panikerkrankungen) einhergehen. Kriterium (3) erfasst psychische Störungen, bei denen die Handlungsfähigkeiten des Betroffenen eingeschränkt sind, z. B. Zwangserkrankungen oder Demenzen. Fortgeschrittene Formen der Demenz erfüllen sogar das Kriterium der Lebensbedrohlichkeit (Kriterium [1]), was natürlich nur zum Tragen kommt, sofern keine entsprechende soziale 
Unterstützung vorhanden ist. Die psychisch bedingte bzw. psychosomatische Impotentia coeundi, z. B. die (psychosomatische) erektile Dysfunktion des Mannes, wird - wie gezeigt - durch das Kriterium (4) abgedeckt.

Es gibt jedoch einige Gruppen als pathologisch betrachteter Verhaltens- und Erlebensweisen, die sich kaum oder gar nicht auf diese Weise rekonstruieren lassen. Bei diesen muss daher die Frage aufgeworfen werden, nach welchem Kriterium oder welchen Kriterien diese Phänomene als krankhaft klassifiziert werden. Um diese Frage eindeutig beantworten zu können, müsste eigentlich geklärt sein, welche Phänomene überhaupt als psychopathologisch gelten sollen - eine Frage, die in der Psychiatrie und Psychopathologie jedoch noch teilweise kontrovers diskutiert wird. Solange diese Kontroversen nicht aufgelöst sind, wird auch jedes vorgeschlagene Krankheitskriterium nicht unumstritten sein. Ich habe daher oben mit der Klausel (5) ein hypothetisches fünftes Krankheitskriterium formuliert, das unter dem Vorbehalt steht, im Zuge einer Einigung über die psychiatrische Krankheitslehre revidiert werden zu können. Allerdings ist die gegebene Formulierung nicht völlig unverbindlich, sondern orientiert sich an dem Kanon psychiatrischer Erkrankungen, die in der Internationalen Klassifikation psychischer Störungen ICD-10, Kap. V (F $)^{13}$ sowie in der letzten Fassung des Diagnostischen und Statistischen Manuals Psychischer Störungen - Textrevision - (DSM-IV-TR $)^{14}$ aufgelistet sind. Diese beiden Systeme haben bekanntlich national und international einen hohen Verbindlichkeitsgrad; die ICD-1o ist in Deutschland die gesetzlich vorgeschriebene Grundlage für die Dokumentation psychiatrischer (und aller anderen) Erkrankungen.

Legt man die in den beiden genannten Handbüchern vorgelegte Krankheitsklassifikation zugrunde, so lassen sich u. a. folgende Krankheitsgruppen als „problematische“ Fälle identifizieren, d. h. als Fälle, in denen die Anwendung der Krankheitskriterien (1) bis (4) nicht möglich oder zumindest nicht vorrangig erscheint (in Klammern der ICD-Code):

- Dissozialität bzw. Antisozialität: erheblich schädigendes oder störendes Verhalten bei fehlendem Leidensdruck und mangelhafter Motivation zur Änderung (F91)

- Persönlichkeitsstörungen: paranoide, dissoziale/antisoziale, anankastische oder ängstlich-vermeidende Persönlichkeitsstörung (F6o.o, 6o.2, $60.5,60.6)$

- Aufmerksamkeitsstörung mit Hyperaktivität, evtl. mit Störung des Sozialverhaltens (ADHS-Syndrom, Fgo.o/Fgo.1)

- Frühkindlicher Autismus/Asperger-Syndrom (F84.0/F84.5).

Dass diese Krankheitsgruppen schwer unter die Krankheitskriterien (1) bis (4) zu bringen sind, sieht man, wenn man die diagnostischen Kriterien betrachtet, die in den genannten Handbüchern angegeben sind. Als Beispiel sei die paranoide Persönlichkeitsstörung zitiert:

13 Vgl. ICD-10 (2005).

14 Vgl. DSM-IV-TR (2003). 
Diagnostische Kriterien für ICD-10 Code F60.0 "Paranoide Persönlichkeitsstörung“:

Mindestens vier der folgenden Eigenschaften oder Verhaltensweisen müssen vorliegen:

1. Übertriebene Empfindlichkeit bei Rückschlägen und Zurücksetzung.

2. Neigung zu ständigem Groll wegen der Weigerung, Beleidigungen, Verletzungen oder Missachtungen zu verzeihen.

3. Misstrauen und eine starke Neigung, Erlebtes zu verdrehen, indem neutrale oder freundliche Handlungen anderer als feindlich oder verächtlich missdeutet werden.

4. Streitsüchtiges und beharrliches, situationsunangemessenes Bestehen auf eigenen Rechten.

5. Häufiges ungerechtfertigtes Misstrauen gegenüber der sexuellen Treue des Ehe- oder Sexualpartners.

6. Tendenz zu stark überhöhtem Selbstwertgefühl, das sich in ständiger Selbstbezogenheit zeigt.

7. Inanspruchnahme durch ungerechtfertigte Gedanken an Verschwörungen als Erklärungen für Ereignisse in der näheren Umgebung und in aller Welt.

Hier ist zu erkennen, dass man zwar versuchen könnte, die Kriterien der Missempfindung oder der (Selbst-)Behinderung heranzuziehen, dass aber das eigentliche Problem anders gelagert ist und eher in der mangelnden ,sozialen Einpassung “ des Betroffenen liegt, d. h. in seiner Unfähigkeit, mit seinen Mitmenschen in einer „normalen“, konfliktfreien Weise zusammenzuleben.

Auch bei dem o. g. Krankheitsbild des Asperger-Autismus geht aus der Definition gemäß ICD-10 bzw. DSM-IV hervor, dass ein Leiden oder eine Behinderung im engeren Sinne nicht vorliegen. Asperger-Patienten können beruflich sehr erfolgreich sein, bis hin zur Tätigkeit als Wissenschaftler. Die eigentliche Störung liegt wiederum in der Unfähigkeit zur „sozialen Einpassung“, d.h. $\mathrm{zu}$ einem adäquaten Verstehen und Beherrschen sozialer Interaktionen und Prozesse. Dies macht die Betroffenen fremdartig für ihre Mitmenschen, isoliert sie und verhindert Kooperation. Man hat ihre soziale Situation einmal bildhaft verglichen mit der eines „Anthropologen auf dem Mars“, also eines Erdmenschen inmitten einer ihm intuitiv völlig unverständlichen Gemeinschaft von Marsmenschen. ${ }^{15}$

Das Gemeinsame dieser beiden Störungen sowie auch der anderen „Problemfälle" bei den psychiatrischen Störungen liegt in der bereits erwähnten „mangelnden sozialen Einpassung“. Dieser Gedanke ist die Crundlage für die Formulierung des fünften Krankheitskriteriums, das spezifisch die psychosoziale und epidemiologische ${ }^{16}$ Pathologie betrifft. Bei der Formulierung ist allerdings zu beachten, dass vermieden werden muss, soziostrukturelle Probleme und Konflikte in einen Betroffenen zu ,introjizieren“: Auch eine Lebensgemeinschaft von Menschen (Familie, Arbeitsteam, Wohngemeinschaft etc.)

15 Vgl. die Titelgeschichte in Sacks (1995).

16 Die epidemiologische Pathologie ist insofern einzubeziehen, als z. B. symptomfreie Überträger von Infektionskrankheiten („carrier“, z. B. bei Salmonellosen) auch unter Kriterium (5) fallen können. Vgl. dazu auch die Kritik von Rosenthal (2007) und meine Replik in Hucklenbroich (2007 b), S. $150 \mathrm{f.}$ 
kann als solche pathologische Züge aufweisen und einen vorher „psychisch gesunden" Menschen zu Verhaltensweisen und Reaktionen zwingen, die dann ihrerseits als ,nicht normal“ und pathologisch imponieren. Man muss daher das Verhalten und Erleben eines Menschen immer auf dem Hintergrund einer als "gesund“ angenommenen Lebensgemeinschaft analysieren und beurteilen, ob er auch in einer solchen gesunden Gemeinschaft pathologisches Verhalten zeigt oder zeigen würde - oder ob sein Verhalten als bloße Reaktion auf eine pathologische soziale Umgebung aufzufassen ist. Das fünfte und letzte Krankheitskriterium ist also sinnvollerweise in Form von zwei Teilkriterien (5a) und ( 5 b) formuliert - einmal bezogen auf das Individuum, einmal bezogen auf das soziale System.

Das Kriterium (5b) würde z. B. zutreffen auf solche Störungen einer Lebensgemeinschaft, wie sie in der „systemischen Familientherapie“ diagnostiziert und behandelt werden. Hierzu würde man auch solche Störungen wie Pubertätsmagersucht (Anorexia nervosa) und sogar Schizophrenie rechnen müssen, wenn die dazu vorgelegten Erklärungsmodelle bestimmter Forschungsrichtungen, z. B. von Stierlin u. a. ${ }^{17}$ für die Anorexia nervosa oder von Bateson, Jackson und Haley ${ }^{18}$ für die Schizophrenie, zutreffend sein sollten. Dies gilt selbst dann noch, wenn man die inzwischen durch biologisch-psychiatrische Forschungen bekannten organischen Prädispositionen für diese Erkrankungen berücksichtigt, da ein wesentliches Merkmal dieser Erkrankungen gerade die Beziehungsstörung ist. Andererseits ist damit nicht ausgeschlossen, dass diese Erkrankungen auch noch nach anderen Kriterien als pathologisch zu klassifizieren sind, z. B. wegen der Lebensgefährdung durch Verhungern bei Anorexie oder wegen des Leidens aufgrund von Angstzuständen bei Schizophrenie.

Das Kriterium (5), insbesondere die Teilklausel (5b), sind in besonderem Maße der Gefahr von Missverständnissen und sinnwidrigem Missbrauch ausgesetzt. Dies hängt damit zusammen, dass die Bereiche von krankhaftem und kriminellem Verhalten eines Individuums sowie krankhafter und gewaltförmiger Verfassung einer Lebensgemeinschaft nicht streng getrennt werden können, sondern immer Überlappungen zeigen werden. Etliche Kritiker plädieren daher dafür, ganz auf dieses Kriterium zu verzichten. Das hätte jedoch den Nachteil, dass die genannten Beeinträchtigungen der Fähigkeit zum sozialen Miteinanderleben ganz aus dem medizinisch-pathologischen Kontext herausfallen würden, obgleich in vielen Fällen enge Beziehungen zwischen organisch-somatischen Gegebenheiten (z. B. Hirnstruktur), Besonderheiten oder Störungen der neuropsychologischen Entwicklung und der psychosozialen Symptomatik selbst nachgewiesen sind. Dies gilt nicht nur für Individuen, sondern kann in Ansätzen auch für Lebensgemeinschaften wie die Mutter-Kind-Dyade oder Familien mit spezifischen Strukturen aufgezeigt werden. ${ }^{19}$ Selbst die Rolle der gesamten umgebenden historisch-sozialen Figuration für die Identitätsbildung - und mögliche Störungen - kann heute mit Mitteln der Hirnforschung

17 Vgl. Weber/Stierlin (1989).

$18 \mathrm{Vgl}$. Bateson/Jackson/Haley (1969).

19 Aus der zahlreichen Literatur sei nur hingewiesen auf einige neuere Übersichtsdarstellungen: Petermann (1998); Herpertz-Dahlmann et al. (2003); Petermann (2004); Fonagy et al. (2004); Förstl (2005), (2007). 
nachgewiesen werden, indem z. B. gezeigt wird, wie bei der Bildung des autobiographischen Gedächtnisses ,aus interpersonalen Beziehungen intrapersonale psychische Formationen werden “. ${ }^{20}$ Die Einbeziehung der in Kriterium (5) genannten Dimensionen in den Krankheitsbegriff steht daher m. E. völlig in der Konsequenz der medizinisch-pathologischen Systematisierung, benötigt aber vielleicht noch mehr erfahrungswissenschaftlich abgesichertes Belegmaterial, ehe eine für alle Diskussionsteilnehmer überzeugende Grundlage gegeben ist. Andererseits würde ich eine Ablehnung dieses Kriteriums, die sich ausschließlich auf die Möglichkeit eines politischen Missbrauchs stützt, für eine Form vorauseilender Unterwerfung wissenschaftlicher Erkenntnis unter politisch-taktische Erwägungen halten, die - zu Ende gedacht - Wissenschaft überhaupt unmöglich machen würde.

\section{Die Konstruktion von Normalität in der Medizin}

Nachdem gezeigt wurde, wie die Medizin Krankhaftigkeit konzeptualisiert, soll nun skizziert werden, wie in diesem Zusammenhang Normalität und Varianz bzw. Abweichung konstruiert werden können. Auch hier handelt es sich um eine Rekonstruktion der Verfahren, die in der Medizin faktisch angewendet werden. Ich betrachte zunächst exemplarisch die Vorgehensweise bei der Etablierung von Normalwerten und Normalbereichen und anschließend das dahinter stehende allgemeine Schema der Konstruktion von Normalität.

\subsection{Die Konstruktion von Normalwerten und Normalbereichen}

Normalwerte bzw. Normal(wert)bereiche sind ein in der ganzen Medizin verwendetes begriffliches Instrumentarium. Ihr Prinzip besteht darin, für Merkmale des Organismus, die entweder diskrete Werte bzw. Ausprägungen annehmen oder kontinuierlich quantitativ variieren können, bestimmte Ausprägungen/ Werte bzw. bestimmte numerische Intervalle anzugeben, die einen Bereich des wahrscheinlich Gesunden von einem Bereich des wahrscheinlich pathologisch Veränderten trennen. Als solche Merkmale oder Parameter kommen grundsätzlich alle am Organismus unterscheidbaren Teile, Eigenschaften und Zustände in Frage, von physikalisch und chemisch definierten Körperbestandteilen über klinisch definierte Funktionen und Vorgänge bis zu psychometrisch definierten Fähigkeiten und Verhaltensdispositionen. Wesentlich für das Verständnis solcher Normalwerte ist die Kenntnis der Art und Weise, wie man zu ihnen gelangt: Wenn für ein bestimmtes Merkmal bzw. einen Parameter die Normalwerte bzw. der Normalbereich ermittelt werden sollen, wird eine möglichst große und repräsentative Stichprobe aus der Population gezogen, für die die Normalwerte/Normalbereiche gelten sollen, und es wird dann die statistische Verteilung des entsprechenden Merkmals/Parameters ermittelt. Solche Verteilungen können unterschiedlich aussehen; in vielen, wenngleich keineswegs in allen Fällen existiert eine sog. Normalverteilung (Gaussverteilung), bei der die Werte/Ausprägungen in bestimmter, mathematisch exakt definierbarer, sym-

20 Vgl. Markowitsch (2005); Welzer (2006); Zitat bei Markowitsch (2005), S. 261. 
metrischer Weise um einen Mittelwert herum gruppiert sind. ${ }^{21}$ Im zweiten Schritt wird aus einer solchen Gesamtverteilung dann ein Bereich ermittelt, der nach bestimmten statistischen Kriterien als „Normalbereich“ gelten darf, insofern eine bestimmter quantitativ festgelegter Anteil der Stichprobe darin enthalten ist. Im Falle einer Normalverteilung besteht ein übliches Verfahren darin, dasjenige Intervall um den Mittelwert als Normalbereich zu wählen, dessen beide Grenzen jeweils um die doppelte Standardabweichung vom Mittelwert abweichen, womit ca. 95,5\% der Stichprobe erfasst werden.

Von grundlegender Bedeutung ist bei diesem Verfahren der Umstand, dass für die Stichprobe nicht eine unausgelesene Population verwendet werden darf, sondern nur eine Population, die ausschließlich aus Gesunden (als gesund Bekannten bzw. Nachgewiesenen) besteht. ${ }^{22}$ Andernfalls bekäme man nicht eine „normale“ Verteilung des untersuchten Merkmals, sondern eine, die durch die Einflüsse verschiedenster Krankheiten in unbekannter Weise verzerrt und verfälscht sein könnte. Dieses Vorgehen impliziert daher insbesondere, dass man bereits über Kriterien verfügen muss, mit denen die Gesunden von den Erkrankten vor der statistischen Erhebung getrennt werden können. Es eignet sich daher auch nicht als primäre Methode zur Definition des Krankheitsbegriffs! Vielmehr stellt es eine Methode dar, unter Voraussetzung eines bereits existierenden primären Krankheitsbegriffs die Unterscheidung zwischen krankhaft und gesund/normal auf (fast) beliebige Merkmale zu erweitern. Dabei wird übrigens in Kauf genommen, dass ein bestimmter Prozentsatz der Gesunden - im obigen Beispiel wären es ca. 4,5\% - als nicht normal bezüglich des untersuchten Wertes eingestuft werden - also als falsch positiv hinsichtlich Erkrankung! Daher darf diese Erweiterung der Krankheitskriterien auch nur als mit einer bestimmten Wahrscheinlichkeit gültig und anwendbar betrachtet werden, wie in der obigen Formulierung bereits berücksichtigt: Wenn ein klinisch Untersuchter einen Wert außerhalb des Normalbereichs aufweist, ist er trotzdem mit einer bestimmten geringen Wahrscheinlichkeit nicht erkrankt, sondern gehört zu den Gesunden, die durch die statistische Methodik artifiziell aus dem Normalbereich ausgeschlossen wurden.

\subsection{Die Unterscheidung von Normalvarianten und pathologischen Abweichungen in der Morphologie}

Das gerade genannte Verfahren der Etablierung von Normalwerten und Normalbereichen eignet sich gut für diskrete oder kontinuierliche quantitative Parameter, dagegen weniger für morphologische Merkmale, also z. B. Varianten in der Form des Körpers oder bestimmter Körperteile, im Vorhandensein oder Fehlen bestimmter Strukturen oder Merkmale oder in der Anzahl oder Größe bestimmter anatomischer Strukturen. Hier wird daher meist ein anderes Vorgehen gewählt, um das Normale vom Pathologischen zu trennen. Dabei wird ebenfalls auf den primären Krankheitsbegriff rekurriert, aber er wird nicht zur Auswahl einer Stichprobe von Gesunden, sondern zur Falldifferenzierung in Anwendung auf Einzelfälle benutzt. Dies sei an zwei einfachen Beispielen demonstriert:

21 Einzelheiten sind den einschlägigen Darstellungen der medizinischen Statistik und Biometrie zu entnehmen. 22 Vgl. z. B. die Darstellung in Löffler/Petrides (2003), S. XXXVII, oder in Thomas (2000), S. 1504. 
Beispiel 1: In der Anatomie des Skelettsystems ist bekannt, dass sich Rippen zwar ganz überwiegend nur im Bereich der Thoraxwirbel finden, in einer kleinen Zahl der Fälle aber auch an den Halswirbeln auftreten können. Man bezeichnet solche Rippen als Halsrippen. ${ }^{23}$ Für solche Halsrippen gilt:

a) Sie treten als Vergrößerungen des Tuberculum anterius des 7. (seltener des 6 . bis 4.) Halswirbels oder als frei bewegliche zusätzliche Rippen auf.

b) Die Stämme des Armnervengeflechts (Plexus brachialis) oder die A. subclavia können durch Halsrippen behindert oder zwischen Halsrippe und 1. Rippe eingeklemmt werden, mit der Folge von Durchblutungs- und Sensibilitätsstörungen.

c) Mögliche Symptome sind Schmerzen und Muskelschwächen im Arm.

Im Hinblick auf Krankhaftigkeit bzw. Krankheitswert von Halsrippen wird daher geurteilt, dass ein Krankheitswert dieser anatomischen Variation nicht generell gegeben ist, sondern nur vorliegt, falls aufgrund ihrer Größe und Lage die in b) oder c) genannten Konsequenzen auftreten. Dann liegt ein aufgrund der Krankheitskriterien (3) (Einschränkung von Muskelkraft und Sensibilität) und (2) (Schmerzen im Arm) als krankhaft zu bewertender Zustand vor. In allen anderen Fällen werden Halsrippen als Normalvariante beurteilt. Diese Beurteilung wird also jeweils im Einzelfall vorgenommen, aber sie orientiert sich an einer Falldifferenzierung, die auf die primären Krankheitskriterien rekurriert.

Beispiel 2: Bei der Untersuchung des Abdomens, z. B. mit Ultraschall oder der Röntgen-Computertomographie, wird häufig als Zufallsbefund eine Variante der Nierenmorphologie gefunden, die als solitäre Nierenzyste bezeichnet wird. Man sieht dann z.B., ausgehend von einer Niere, eine mehr oder weniger große homogene Verschattung mit geringer Röntgendichte, die im Extremfall fast den gesamten ipsilateralen Bauchraum ausfüllen kann. Für solche Solitärzysten gilt: ${ }^{24}$

a) Sie sind entstanden durch eine Entwicklungsstörung, bei der Nierenkanälchen und Ast des Sammelrohrs nicht zusammenfinden. Der im zugehörigen Nierenkörperchen gebildete Primärharn kann nicht abfließen und staut sich an. Es entsteht ein Hohlraum, der bis auf Fußballgröße heranwachsen kann.

b) Sie können wie eine Geschwulst die Nachbarorgane verdrängen, die Arbeit der Niere beeinträchtigen (Kompression des ableitenden Harnsystems) und Flankenschmerzen verursachen, wenn die Zyste infolge ihrer Cröße die Nachbarorgane stört.

c) Außerdem kann es zu Komplikationen wie Ruptur, Infektion, Einblutung oder maligner Entartung kommen, durch die sekundär Schmerzen, Schwäche und sogar lebensbedrohliche Zustände auftreten können.

23 Vgl. Benninghoff/Drenckhahn (2003), S. 424-426; dort auch Beispiele für weitere Variationen und Fehlbildungen.

24 Zu Ätiologie, Pathogenese, Morphologie und Krankheitswert von Nierenzysten vgl. Böcker (2004), S. 835-836, und Riede (2004), S. 810-813. 
Im Hinblick auf den Krankheitswert von solitären Nierenzysten ist ebenfalls zu sagen, dass dieser keineswegs generell gegeben ist bzw. angenommen wird. Solitäre Nierenzysten gelten als primär gutartige Variationen der „normalen“ Entwicklung, die jedoch dann einen Krankheitswert erhalten können, wenn aufgrund ihres Wachstums oder einer akut eintretenden Veränderung die in b) und c) genannten Komplikationen auftreten. Nur dann liegt nach den Kriterien (3) (Einschränkung der Nierenleistung), (2) (z. B. Flankenschmerz) und (1) (Lebensbedrohlichkeit) ein als pathologisch zu beurteilender Zustand vor. Festzuhalten ist also: Obwohl eine solitäre Nierenzyste keine (positive) Funktion im Organismus erfüllt und ihre ätiologische Erklärung sogar einen Vorgang zugrundelegt, der als „Störung“ oder „Fehler“ bezeichnet werden kann, wird sie solange als Normalvariante eingestuft, wie sie keine expliziten Krankheitskriterien erfüllt.

An diesen beiden Beispielen wird die generelle Strategie der Klassifikation morphologischer Varianten erkennbar: Maßgeblich dafür, unter den vorkommenden Variationen der Morphologie die normalen von den krankhaften zu unterscheiden, ist für die Medizin nicht deren Häufigkeit bzw. Seltenheit, nicht einmal deren vorhandene oder fehlende Funktionalität, sondern ausschließlich die Frage, ob explizit Krankheitskriterien erfüllt sind oder nicht. Davon muss deutlich die Beurteilung nach ästhetischen Kriterien unterschieden werden, die z.B. bestimmte Variationen von Körperform, Hautfärbung und Behaarung oder das Vorkommen von bestimmten Hautanhangsgebilden und (gutartigen) Tumoren als Abweichung von gesellschaftlich etablierten ästhetischen Idealen oder Normen wertet - und daraus möglicherweise eine ästhetische bzw. kosmetische Behandlungsindikation ableitet.

\section{Die Konstruktion von Normalität: Probleme}

\subsection{Deskriptivität und Normativität}

Die in den beiden vorhergehenden Abschnitten dargestellten Methoden zur Konstruktion von Normalität lassen ein allgemeines Paradigma für die Medizin erkennen: Wenn die Fälle ausgesondert sind, die sich gemäß dem Krankheitsbegriff eindeutig als pathologisch identifizieren lassen, ist der verbleibende gesamte übrige Spielraum als Variationsbreite des Normalen aufzufassen. Seltenes Vorkommen und fehlende Funktionalität sind keine Ausschlusskriterien für Normalität, ebenso wenig wie bloße Abweichung von ästhetischen Idealen oder von kulturell konstruierten (z. B. sportlichen) Leistungsnormen. Dagegen können durchaus Zustände oder Merkmale, die aus kultur- oder gesellschaftsgebundenen Vorstellungen heraus als normal oder sogar als positiv gelten, in medizinischer Sicht als pathologisch zu werten sein. Man denke nur an die Lotusfüßchen in der chinesischen Kultur, die Giraffenhalsfrauen in Thailand, den Embonpoint und die Wespentaille im 19. Jahrhundert und die Twiggy- bzw. Supermodel-Figur im 20. Jahrhundert in der westlichen Kultur. Daran zeigt sich, dass der medizinische Normalitätsbegriff und die mit ihm gemachten Unterscheidungen nicht einfach mit dem allgemeinen, lebensweltlich gebrauchten Begriff der Normalität identifiziert werden können. Der wichtigste Unterschied ergibt 
sich daraus, dass sowohl der Krankheits- als auch der Normalitätsbegriff in der Medizin als Begriffe einer wissenschaftlichen Theorie verstanden werden. Welche Konsequenzen das hat, soll etwas ausführlicher dargestellt werden:

Die im vorigen Kapitel dargestellte Rekonstruktion des Krankheitsbegriffs diente dazu, die Unterscheidung zwischen normal und krank (bzw. krankhaft) so nachzuzeichnen, wie sie im Rahmen der medizinischen Krankheitslehre verwendet wird. Ich hatte bereits darauf hingewiesen, dass sich diese Unterscheidung nicht mit der gleich lautenden alltäglichen, vorwissenschaftlichen Unterscheidung deckt. Der entscheidende Unterschied besteht darin, dass sich die wissenschaftlich-medizinische Unterscheidung nur als eine deskriptive Unterscheidung verwenden lässt, jedenfalls dann, wenn sie in Übereinstimmung mit der rekonstruierten Definition gebraucht wird: Wenn gemäß der gegebenen Definition zwischen normal und krankhaft unterschieden wird, hängt das Ergebnis der Unterscheidung nur davon ab, welche Sachlage gegeben ist, d.h. ob eines oder mehrere der Definitionskriterien erfüllt sind oder nicht es hängt dagegen nicht davon ab, welche wertende (evaluative) Einstellung der Beurteiler gegenüber dieser Sachlage oder gegenüber dem allgemeinen Phänomen „Krankheit“ einnimmt, oder welche Handlungskonsequenzen (normativen Folgerungen) er aus dem gegebenen Sachverhalt, oder allgemein aus dem Vorliegen von Krankheit, zu ziehen beabsichtigt. Die Deskriptivität des Krankheitsbegriffs ist mithin ein Spezialfall desjenigen Merkmals, das in der Methodologie üblicherweise als Objektivität (Unabhängigkeit vom Beobachter) bezeichnet wird. Diese Deskriptivität folgt daraus, dass die Kriterien für die Beurteilung eines Phänomens als krankhaft oder normal in ihrem Inhalt durch die gegebene Definition explizit festgelegt sind und insofern keinen Spielraum für die Einbeziehung subjektiver Wertungen des Beurteilers lassen; eine Einbeziehung subjektiver Wertungen kann, sobald sie das Ergebnis der Unterscheidung überhaupt tangiert, nur eine Verfälschung dieses Ergebnisses sein. Einige Beispiele zur Erläuterung: Wer z. B. aus Stolz oder Scham leugnet, einen tatsächlich empfundenen Schmerz zu verspüren („Das tut mir überhaupt nicht weh!“), verfälscht die Tatsachen; wer einem anderen -z. B. aus Rechthaberei die Anerkennung des zugefügten und subjektiv angegebenen Schmerzes verweigert („Das kann Ihnen doch gar nicht weh getan haben!“), verleugnet ebenfalls die Wahrheit; wer von einem malignen, die Lebenserwartung verkürzenden Prozess betroffen ist, dies aber nicht als krankhaft bezeichnen will, weil er des Lebens ohnehin überdrüssig ist, hat zwar das Recht auf Behandlungsverzicht, verfehlt aber die medizinische Definition von Krankhaftigkeit.

In diesem Sinne ist die medizinische Unterscheidung deskriptiv, d. h. rein beschreibend und von Werten und Normen unabhängig. Dieser Sachverhalt wird prinzipiell auch dadurch nicht berührt, dass natürlich keine Definition unendlich genau und exakt, und nicht jede konkrete Sachlage absolut eindeutig bestimmt ist: Auch wenn aufgrund von Ungenauigkeiten oder Uneindeutigkeiten subjektive Einstellungen des Beurteilers in die Unterscheidung einfließen, wird dadurch die prinzipielle Intention, mit der Unterscheidung eine bestehende Sachlage $\mathrm{zu}$ beschreiben und nicht ein subjektives Werturteil zu äußern, nicht desavouiert, sondern es wird lediglich ein nicht vermeidbarer subjektiver Ermessensspielraum ausgefüllt. In solchen Fällen kann es dann allerdings - im Rah- 
men des Ermessensspielraums - zu divergierenden, von Werthaltungen abhängigen Beurteilungen kommen; daraus folgt aber nicht die Wertabhängigkeit des zugrunde liegenden Begriffs selbst. Die Sachlage ist also im Fall des medizinischen Krankheitsbegriffs grundsätzlich anders als etwa bei ästhetischen, politischen oder moralischen Beurteilungskategorien: Ob z. B. ein Kinofilm ein schöner Film, eine politische Entscheidung eine richtige Entscheidung und eine Handlung eine moralisch gute Handlung ist, lässt sich nicht ohne Rekurs auf Werte, Normen, Einstellungen oder Geschmacksrichtungen unterscheiden, und daher sind diese Unterscheidungen nicht rein deskriptiv.

Es wird oft zur Verteidigung der Normativität des Krankheitsbegriffs das Argument ins Feld geführt, der Krankheitsbegriff diene zur Begründung von Entscheidungen normativer Art, insbesondere Entscheidungen über Behandlung oder Nichtbehandlung, bzw. über Behandlungsbedürftigkeit; deswegen müsse der Begriff mindestens auch normative oder evaluative Komponenten enthalten. Dieses Argument ist falsch und beruht auf einer ungenügenden logischen Analyse von Entscheidungen der genannten Art. Die Tatsache, dass ein Begriff bei der Begründung von Entscheidungen eine wesentliche Rolle spielt, bedeutet mitnichten, dass dieser Begriff selbst normative Bedeutungskomponenten aufweisen muss. Das kann man ganz einfach an folgender Tatsache sehen: Es ist durchaus denkbar, dass die Entscheidung über die Behandlungsbedürftigkeit bestimmter Zustände z. B. vom Lebensalter des Betroffenen abhängig gemacht wird. Nun ist der Begriff Lebensalter aber sicherlich ein rein deskriptiver Begriff, der nur von der tatsächlich gelebten Zeitdauer und nicht von Werten oder Normen des Beurteilers abhängig ist. Trotzdem kann ein solcher deskriptiver Begriff als Kriterium bei der Anwendung von Normen zur Begründung von Entscheidungen fungieren. Dies gilt auch für den Krankheitsbegriff: Er fungiert häufig (aber keineswegs immer) als Kriterium bei der Entscheidung, ob ein bestimmter Zustand behandelt werden soll, ohne dazu selbst normative Bedeutungskomponenten enthalten zu müssen. Die Normativität ist vielmehr in den expliziten Normen zu finden, auf denen solche Entscheidungen beruhen, wenn z. B. die Norm zugrunde gelegt wird: ,Jeder krankhafte Zustand sollte prinzipiell ärztlich behandelt werden. " ${ }^{25}$ (Ob diese Norm eine gute Norm wäre, soll hier nicht weiter diskutiert werden.)

Ein Nebenergebnis dieser Argumentation ist, dass die Definition des Krankheitsbegriffs weiterhin in die Zuständigkeit der Medizin fällt und nicht etwa in die der Medizinethik - was u. a. von Wolfgang Wieland und Urban Wiesing behauptet wurde: ${ }^{26}$ Sie wollen aus der Tatsache, dass der Krankheitsbegriff innerhalb normativer Entscheidungen benutzt wird, eine Art „Mitspracherecht“ der Medizinethik bzw. des Medizinethikers bei der Unterscheidung zwischen normal und krank(haft) ableiten. Wiesing geht sogar so weit, der Medizin das Recht zur Definition dieser Unterscheidung ganz zu bestreiten und sie vollständig der Zuständigkeit „moralischer Argumente“ und damit der (Medizin-)

25 So auch Hoffmann (2006), S. $209 \mathrm{ff}$ - Eine ausführlichere Analyse der logischen Ebenen und Schritte bei Entscheidungen über ärztliche Behandlung habe ich gegeben in Hucklenbroich (2006), Kap. 5.2.

26 Wieland (1986), S. 35-41, und (2004), S. 19-28; Wiesing (1998), (2004), (2007). Vgl. schon meine Kritik in Hucklenbroich (1998). 
Ethik zu unterstellen. Diese kuriose Position, die die Ethik völlig überfordern würde, beruht auf einer simplen Verwechslung: Was die Medizinethik zuständigerweise untersuchen und ggf. begründen kann, ist nicht der Krankheitsbegriff, sondern sind die Normen, die bei Behandlungsentscheidungen zugrunde gelegt werden und in denen der Krankheitsbegriff, aber auch andere Kriterien lediglich herangezogen - aber nicht definiert - werden. ${ }^{27}$ Die Analyse und Rekonstruktion der Art und Weise, wie der Krankheitsbegriff in der Medizin definiert und gebraucht wird, ist dagegen eine typische wissenschaftstheoretische Aufgabe und fällt daher nicht in das Gebiet der Medizinethik, sondern der Theorie der Medizin bzw. in deren Teilgebiet, die Theoretische Pathologie. So auch der Medizinhistoriker Heinrich Schipperges mit Blick auf die Position Wielands und Wiesings:

„Der Wissenschaftshistoriker tut sich zunächst einmal recht schwer bei seiner Suche nach einem gängigen, einigermaßen verbindlichen Krankheitsbegriff. Ein Krankheitsbegriff sollte selbstverständlich alle Erscheinungen von „krank“ vom Phänomen „nicht krank“ abgrenzen und für beide Felder die zureichenden Merkmale liefern. [...] Nun muß sicherlich zugegeben werden, daß Krankheitsbegriffe wie Krankheitsvorstellungen überhaupt einem historischen Wandel unterliegen und vom jeweiligen soziokulturellen Kontext abhängig bleiben. Daher wird man auch - bei allen noch so pragmatischen Rechtfertigungen - auf historisch gewachsene Krankheitsvorstellungen und Lebensentwürfe zurückgreifen müssen. In diesem Rahmen aber bleibt die Analyse des Krankheitsbegriffs eher die Aufgabe einer , Theoretischen Pathologie' als die einer ,Medizinischen Ethik'. “28

Missverständnisse, wie sie den hier kritisierten (und ähnlichen) Positionen zugrunde liegen, die eine Normativität oder Werthaftigkeit des Krankheitsbegriffs annehmen, können allerdings dadurch verursacht sein, dass deren Vertretern eigentlich nicht der medizinische, sondern der außerwissenschaftliche, lebensweltliche Krankheitsbegriff vor Augen steht. Es ist ja nicht zu bestreiten, dass im alltäglichen Leben Krankheit meistens - wenn auch keineswegs immer - ein unerwünschtes, ja gefürchtetes Ereignis darstellt und in den Bezeichnungen Krankheit, krank und krankhaft daher diese negative Wertung in der Regel mitschwingt und mit zum Ausdruck gebracht werden soll. Der lebensweltliche Krankheitsbegriff darf deswegen, soweit man ihn überhaupt fixieren kann oder will, als „wertbehafteter“ Begriff bezeichnet werden - wobei man aber berücksichtigen muss, dass es auch in der Lebenswelt Kontexte und Situationen gibt, in denen Krankheit nicht negativ, sondern neutral oder sogar positiv gewertet wird und trotzdem als „Krankheit“ bezeichnet wird; die Alltagssprache ist offenbar so elastisch, dass sie für solche Fälle auch einen wertfreien Krankheitsbegriff zur Verfügung hat. Wegen dieser Flexibilität der Alltagssprache macht es allerdings wenig Sinn, aus ihr eine philosophische Grundlagenentscheidung ableiten zu wollen.

27 Dass Wiesings Position, wenn sie beim Wort genommen wird, sogar auf einen logischen Widerspruch hinausläuft, habe ich gezeigt in Hucklenbroich (2007 b), S. $155 \mathrm{f}$.

28 Schipperges (1999), S. 18-20 (Hervorhebung von mir). 


\subsection{Lebensweltliche Normalitätsvorstellungen und medizinische Kriterien}

In der Lebenswelt außerhalb der wissenschaftlichen Medizin i. e. S. wird unter Normalität die Übereinstimmung mit den Normen verstanden, die in einer Gesellschaft/Kultur oder Subkultur ,akzeptiert“ sind und als selbstverständlich gelten. Solche Normen beziehen sich sowohl auf körperliche und seelische Eigenschaften als auch auf Verhaltensweisen und Lebensformen. Abweichungen von diesen Normen werden unterschiedlich konzeptualisiert, z. B. als Missgestalt (Missbildung), Abartigkeit (Perversität), Delinquenz oder politische bzw. ästhetische Provokation, aber auch als Krankheit bzw. Krankhaftigkeit (dann entgegen der medizinischen Bedeutung). Entsprechend unterschiedlich sind die gesellschaftlichen Reaktionen darauf, von Hospitalisierung, Ausgrenzung, Diskrimierung (Stigmatisierung), Kriminalisierung, Verfolgung (Strafverfolgung) bis zur Tolerierung und Einweisung in spezifische Reservate. ${ }^{29}$ In medizintheoretischer Hinsicht sind in diesem Zusammenhang zwei Feststellungen zu machen, ohne auf das Phänomen des gesellschaftlichen Umgangs mit solchem Anderssein umfassend eingehend zu können:

Erstens ist es wichtig zu unterscheiden, ob die lebensweltliche Kategorisierung eines Phänomens als krankhaft von der medizinischen Definition des Krankheitsbegriffs gedeckt wird oder sich ausschließlich außermedizinischen Normalitätsvorstellungen verdankt. Was von diesen beiden Möglichkeiten der Fall ist, kann nicht dadurch entschieden werden, ob der Beurteilende professionell der Medizin bzw. dem Gesundheitssystem angehört oder nicht: Es lassen sich genügend Beispiele in Geschichte und Gegenwart finden, wo auch Ärzte, sogar führende medizinische Autoritäten, sich im Irrtum hierüber befunden haben bzw. sich durch weltanschaulich oder ideologisch begründete Normen statt durch medizinisch begründbare Kriterien haben bestimmen lassen. Wissenschaftlich vertretbar und ärztlich verantwortbar ist die Einschätzung eines Phänomens als krankhaft aber nur dann, wenn sie ihre Kriterien explizit ausweisen kann und damit zu erkennen gibt, ob sie medizinisch oder außermedizinisch motiviert ist. Solange diese Kriterien nicht klar erkennbar sind, darf bezweifelt werden, ob es sich um eine wissenschaftlich-medizinische Beurteilung handelt.

Zweitens, und im nur scheinbaren Gegensatz zum soeben Gesagten, muss festgehalten werden, dass auch bei Zuständen und Erscheinungen, die im medizinischen Sinn nicht krankhaft sind, eine ärztliche Behandlung (z. B. Korrektur) trotzdem indiziert und ethisch gerechtfertigt sein kann - gerade auch dann, wenn es sich um Zustände handelt, die (nur) im außermedizinischen Sinn als krankhaft oder nicht normal gelten! Dies ist typischerweise dann der Fall, wenn die ärztliche Behandlung dem Schutz des Betroffenen vor nachteiligen gesellschaftlichen Reaktionen dient, die aus der herrschenden Normalitätsvorstellung resultieren würden. Zu denken ist an Hänseleien, Quälereien oder Mobbing durch Gleichaltrige, denen Kinder mit auffälligen körperlichen Merkmalen ausgesetzt sein können, aber auch an ästhetisch als fremdartig oder abstoßend bewertete Merkmale bei Erwachsenen. Die ethische Einschät-

29 Die klassische soziologische Abhandlung zum Thema der Stigmatisierung ist Goffman (1967). 
zung solcher korrigierender Eingriffe ist teilweise kontrovers, insofern darin eine „Komplizenschaft“ des Arztes mit der benachteiligenden Gesellschaft oder eine unzulässige „Medikalisierung“ nicht krankheitsbedingter Phänomene gesehen wird. Dieser ethischen Diskussion kann ich hier nicht nachgehen. Es dürfte aber auch für die Gegner solcher korrigierender Eingriffe Fälle geben, in denen sie eine ärztliche Behandlung für gerechtfertigt halten. Aus medizintheoretischer Sicht ist das Besondere an solchen Behandlungen, dass sie im strengen Sinn keine Behandlung von Krankheiten sind, selbst dann nicht, wenn die herrschende gesellschaftliche Ansicht den zu korrigierenden $\mathrm{Zu}$ stand als krankhaft betrachtet. Zur Rechtfertigung solcher Behandlung ist daher auf Normen zurückzugreifen, die eine Behandlungsindikation auf andere Ziele als die Beseitigung von Krankheiten stützen, z. B. auf den Schutz vor gesellschaftlicher Benachteiligung oder Schädigung. Es handelt sich dabei also um Spezial-Indikationen, die nicht oder zumindest nicht ausschließlich auf den Krankheitsbegriff rekurrieren - ähnlich wie im Fall der Behandlung von altersbedingten Beschwerden. Auch die Empfängnisverhütung oder künstliche Sterilisierung ist übrigens eine Behandlung und/oder Prävention nicht krankhafter Zustände. Im Falle der Schutz-Indikation kann allerdings grundsätzlich diskutiert werden, ob die schädigende Lebensgemeinschaft oder Gesellschaft nicht nach Kriterium ( 5 b) als krank zu beurteilen ist - was einerseits nicht $a$ priori ausgeschlossen werden sollte, andererseits aber erst noch durch die Entwicklung einer methodisch ausgearbeiteten Sozialpathologie wissenschaftlich abzusichern wäre. Dies gilt übrigens unbeschadet der Möglichkeit einer (zusätzlichen) ethischen oder politischen Bewertung solcher sozialer Prozesse.

Zusammenfassend möchte ich festhalten,

- dass die medizinische Konstruktion von Normalität auf dem Krankheitsbegriff aufbaut und diesen mit fallvergleichenden und statistischen Methoden erweitert, ohne zusätzliche inhaltliche oder bewertende Kriterien heranzuziehen;

v dass der medizinische Krankheits- und Normalitätsbegriff in dem Sinne als deskriptiv zu bezeichnen ist, dass die korrekte Anwendung der Krankheitskriterien nicht durch subjektive Wertungen des Beurteilers beeinflusst sein kann und normative Konsequenzen nicht aus diesen Begriffen und Kriterien allein, sondern nur unter expliziter Heranziehung normativer Sätze gezogen werden können;

- dass medizinische und gesellschaftliche Normalitätsvorstellungen nicht zusammenfallen (müssen);

- dass gesellschaftliche Vorstellungen von Normalität und Krankhaftigkeit bestimmte ärztliche Maßnahmen notwendig machen können, die keine Krankheitsbehandlung sind, die aber durch Spezial-Indikationen (insbesondere die Schutz-Indikation) normativ begründet werden können. 


\section{Stufen der Allgemeinheit in der Krankheitstheorie und die Frage der Historizität des Krankheitsbegriffs}

Die medizinische Krankheitslehre hat sich in einer langen geschichtlichen Entwicklung herausgebildet und hat bis zum Erreichen des heutigen Standes mannigfache Formen durchlaufen. Diese Geschichte ist von Medizinhistorikern immer wieder dargestellt worden, häufig unter Bezeichnungen wie „Geschichte des Krankheitsbegriffs“. ${ }^{30}$ Dieser Sprachgebrauch kann dazu verführen, von einer Geschichtlichkeit des Krankheitsbegriffs selbst im Sinne seiner historischen Relativität, ohne jede Differenzierung, auszugehen und zu sprechen. Als Beleg für eine solche historische Relativität wird dann gern der historisch zu beobachtende Wechsel in der Einschätzung bestimmter Phänomene als normal oder krankhaft angeführt. Zum Beispiel wird in diesem Zusammenhang das Argument vorgebracht, der Wechsel in der Einschätzung von Homosexualität, Alkoholismus und Säuglingssterblichkeit gehe auf einen Wechsel des Krankheitsbegriffs zurück oder beweise diesen..$^{31}$ Diese Schlussfolgerung ist jedoch erstens schon in dieser Form logisch nicht zwingend: Statt eines Wechsels im Krankheitsbegriff kann, logisch gesehen, genauso gut ein Wechsel im Verständnis des jeweiligen Phänomens zugrunde liegen, durch den das Phänomen nunmehr entweder aus dem Krankheitsbegriff herausfällt, oder aber erstmals unter ihn subsumierbar wird. Dabei wäre der Krankheitsbegriff gerade der historisch konstant bleibende Bezugspunkt, so dass man nicht von einem Wechsel oder Wandel dieses Begriffs sprechen könnte. Zweitens muss aber betont werden, dass die ganze Betrachtungsweise zu undifferenziert bleibt, wenn man nur den ganz allgemeinen Krankheitsbegriff mit den konkreten fraglich krankhaften Phänomenen ins Verhältnis setzt. Es muss hier vielmehr berücksichtigt werden, dass in der Krankheitslehre mehrere Ebenen der Konzeptualisierung von Krankheit zu unterscheiden sind, die unterschiedliche Allgemeinheits- oder Abstraktionsstufen repräsentieren. Diese unterschiedlichen Ebenen sind auch unterschiedlich von den Phänomenen des historischen Wandels betroffen:

Die allgemeinste Ebene in der Krankheitslehre wird von der Krankheitsdefinition dargestellt (oben in D1 und D2 zusammengestellt). Diese Ebene darf als konstitutiv für die westliche (abendländische), naturalistische und erfahrungsbasierte (= „rationale“ bzw. wissenschaftliche) Form von Medizin und Krankheitslehre überhaupt angesehen werden. Abweichungen auf dieser Ebene, also die Verwendung anderer als der in der Definition genannten Bedingungen und Kriterien, liegen historisch in solchen Kulturen vor, in denen die Funktionen von Arzt, Priester, Richter und Prophet (noch) nicht vollständig voneinander getrennt sind, in denen also z. B. schuldhaftes Verhalten (wie z. B. ein Tabubruch), Naturereignisse wie z.B. Dürreperioden und politische Ereignisse, wie z. B. Sieg oder Niederlage im Krieg, in die Zuständigkeit des Heilers fallen können. Wir sollten nicht sagen, dass in diesen Kulturen ein anderer Krankheitsbegriff vorliegt, sondern dass noch gar kein eigentlicher

$30 \mathrm{Vgl}$. exemplarisch Berghoff (1947).

31 So z. B. Wieland (1986), S. 36-39; (2004), S. 24-25; (2007), S. 135. - Zur Kritik vgl. Hoffmann (2006). 
(rationaler) Krankheitsbegriff ausdifferenziert wurde: Die Begriffe von Krankheit, Verbrechen (gesellschaftlich sanktioniertem Verhalten), Naturkatastrophen und gesellschaftlichem Konflikt sind noch nicht deutlich geschieden, sie existieren noch nicht als eigenständige Begriffe; an ihrer Stelle stehen andere, „primitivere“ Vorstellungen, die mit den jeweiligen mythischen und religiösen Glaubensvorstellungen assoziiert sind. Wenn in diesen Vorstellungen ein Wandel eintritt (wie vermutlich in der griechischen Antike bei den ionischen Naturphilosophen und den hippokratischen Ärzten), sprechen wir besser nicht von einem Wandel im Krankheitsbegriff, sondern von der erstmaligen Entstehung eines rationalen Krankheitsbegriffs.

Eine Stufe unterhalb der Krankheitsdefinition liegt das Rahmenmodell für die Erklärung natürlicher Lebens- und Krankheitsvorgänge: Welche Bestandteile den menschlichen Organismus ausmachen, wie sie miteinander interagieren und welche generellen Ursachen jeweils für normale und pathologische Abläufe namhaft zu machen sind, gehört zum allgemeinen Erklärungsrahmen. Innerhalb dieses Rahmenmodells ist die jeweilige Auffassung des Verhältnisses somatischer und psychischer „Anteile“ am Organismus, also die jeweilige Leib-Seele-Theorie, ein besonders wichtiger Teilaspekt. Das Rahmenmodell hat sich im Laufe der Medizingeschichte von der antiken Humoralpathologie bis zum heutigen bio-psycho-sozialen Modell mehrfach verändert, wobei als die wichtigste Zäsur üblicherweise der Anschluss der Medizin an die Theorien, Begrifflichkeiten, Denk- und Forschungsmethoden der neuzeitlichen Erfahrungswissenschaften im ersten Drittel des 19. Jahrhunderts gesehen wird.

Innerhalb des jeweiligen Rahmenmodells und nochmals eine Allgemeinheitsstufe darunter sind die spezifischen erklärenden Theorien für ganz bestimmte Zustände und Abläufe im Organismus anzusiedeln, insbesondere für die Funktion von Organen und Körperbestandteilen, die Mechanismen von Veränderungen und Entwicklungsvorgängen und die Verursachung, Entstehung und Entwicklung krankhafter Abläufe. Dies ist, im gegenwärtigen Rahmenmodell, der Bereich der allgemeinen und speziellen Pathologie, Pathophysiologie und Psychopathologie. Parallel dazu, also auf derselben Allgemeinheitsstufe, ist das System nosologischer Entitäten anzusetzen, also die Systematik und Einteilung der Krankheitsentitäten, die sich auf der Basis klinischer Erfahrung und unter Einbeziehung des Wissens der pathologischen Disziplinen ergibt.

Betrachtet man nun die Veränderungen, die sich im Lauf der Geschichte der Krankheitslehre bezüglich der Einordnung bestimmter Phänomene ergeben haben, so zeigt sich, dass wir es mit zwei großen Gruppen zu tun haben: Die erste, weitaus größere Gruppe ist die derjenigen Veränderungen, die sich aus Entdeckungen und theoretischen Innovationen im Bereich der erklärenden Theorien und der klinischen Erfahrung sowie aus den großen Verschiebungen der Rahmenmodelle ergeben. Unterschiedliche Auffassungen über ursächliche Zusammenhänge, über Funktion und Nutzen körperlicher Vorgänge oder über den Leib-Seele-Zusammenhang führen zu unterschiedlicher Bewertung hinsichtlich der Gesundheit oder Pathologizität der fraglichen Vorgänge. Dabei wird die Definition von Krankhaftigkeit nicht verändert, sondern diese ist gerade diejenige Instanz, auf die man sich bei der Neubewertung letztlich bezieht. Die Neubewertung der Säuglingssterblichkeit beispielsweise ergibt sich 
gerade aus der Entdeckung spezifischer Todesursachen, die nicht notwendig und unvermeidlich aus der Gesetzmäßigkeit körperlicher Entwicklung resultieren, sondern akzidentell sind und somit eine natürliche Alternative besitzen. Wenn es aber eine nicht letal verlaufende natürliche Alternative gibt, ist per definitionem der Tod vorzeitig und somit pathologisch! In dieser Weise lässt sich bei den meisten Veränderungen aufzeigen, dass sie keine Änderung des Krankheitsbegriffs sind, sondern diesen gerade als konstant voraussetzen.

Eine kleine zweite Gruppe von Veränderungen bzw. Differenzen kann man so beschreiben, dass eine fehlerhafte Anwendung des Krankheitsbegriffs bzw. eine Inkonsequenz bei seiner Anwendung aufgedeckt wird. Diese Form der Differenz liegt beispielsweise vor, wenn die Einordnung eines Phänomens und Verhaltens als krankhaft eigentlich auf nicht-medizinischen Motiven beruht, z. B. religiösen, moralischen oder ästhetischen Vorstellungen, diese Bewertung aber - inkorrekt - als medizinische Erkenntnis ausgegeben wird. Insbesondere auch als Folgen christlich-religiöser Moralvorstellungen lassen sich solche Fehleinordnungen historisch aufzeigen. Bei der Aufdeckung und Korrektur solcher Fehlauffassungen liegt nicht etwa ein Wechsel im Krankheitsbegriff vor, sondern es wird eine Inkonsequenz in der Anwendung des rationalen Krankheitsbegriffs, eine Vermischung von medizinischen mit z. B. religiösen Vorstellungen aufgezeigt. Letzteres kann auch dann der Fall sein, wenn diese Klassifikation von Medizinern vorgenommen wurde - deren Urteil ist genauso wenig sakrosankt wie jedes andere Urteil und muss sich an unpersönlichen Kriterien messen lassen. Eine Kritik und Korrektur solcher Fehler als einen Wechsel im medizinischen Krankheitsbegriff zu betrachten, wäre hier eine Verkennung der tatsächlichen Zusammenhänge.

In den Fällen, die üblicherweise für eine historische Relativierbarkeit bzw. Variabilität des Krankheitsbegriffs ins Feld geführt werden, wie z. B. die drei oben genannten, liegt nachweislich der Sachverhalt vor, dass die Klassifikation gemäß dem allgemeinen rationalen Krankheitsbegriff nur zu einem einzigen, eindeutig bestimmten Resultat führt: Homosexualität ist eindeutig nicht pathologisch (außer wenn sie als Folge einer Erkrankung auftritt), Alkoholismus (im Sinne einer Substanz-Abhängigkeit) ist eindeutig krankhaft, und der Tod im Säuglingsalter ist immer ein vorzeitiger Tod und geht immer auf pathologische bzw. pathogene Ereignisse oder Bedingungen zurück.

Es wäre eine lohnende Aufgabe der medizinhistorischen Forschung, die in der Geschichte solcher Klassifikationen vorkommenden Abweichungen vom Krankheitsbegriff und methodologischen Inkorrektheiten einmal detailliert aufzudecken und als Fehler oder medizinische Inkonsequenzen kenntlich zu machen. (Dies ist natürlich nicht zu verwechseln mit dem Versuch, früheren Zeiten in anachronistischer Weise heutige Maßstäbe zu supponieren - es geht um Fehler, die auch damals schon welche waren.) Dann würde sich die Auffassung, dass dem Wandel der Einschätzung bestimmter Lebenserscheinungen notwendigerweise eine historische Änderung und Variabilität des Krankheitsbegriffs zugrunde liege, jedenfalls nicht mehr als so selbstverständlich präsentieren lassen. 


\section{Literatur}

Bateson/Jackson/Haley (1969): Gregory Bateson, Don D. Jackson, Jay Haley, Schizophrenie und Familie, Frankfurt a. M. 1969

Bauer (2007): Axel W. Bauer, Brute Facts oder Institutional Facts? Kritische Bemerkungen zum wissenschaftstheoretischen Diskurs um den allgemeinen Krankheitsbegriff, Erwägen - Wissen - Ethik 18 (2007), 1, S. 93-95

Becker/Doerr/Schipperges (1993): Volker Becker, Wilhelm Doerr, Heinrich Schipperges, Krankheitsbegriff und Krankheitsforschung im Lichte der Präsidialansprachen der Deutschen Gesellschaft für Pathologie (1897-1992), Stuttgart 1993

Benninghoff/Drenckhahn (2003): Alfred Benninghoff, Detlev Drenckhahn (Hrsg.), Anatomie, Bd. 1, München, Jena, 16. Aufl., 2003

Berghoff (1947): Emanuel Berghoff, Entwicklungsgeschichte des Krankheitsbegriffes, Wien, 2. Aufl., 1947

Böcker/Denk/Heitz (2004): Werner Böcker, Helmut Denk, Philipp U. Heitz (Hrsg.), Pathologie, München, Jena, 3. Aufl., 2004

Boorse (1976): Christopher Boorse, On the distinction between disease and illness, Philosophy and Public Affairs 5 (1975), p. 49-68

Boorse (1977): Christopher Boorse, Health as a theoretical concept, Philosophy of Science 44 (1977), p. 542573

Boorse (1997): Christopher Boorse, A Rebuttal on Health, in: James M. Humber, Robert F. Almeder (ed.), What is Disease?, Totowa 1997, p. 3-134

Buyx/Hucklenbroich (2007): Alena Buyx, Peter Hucklenbroich, „Wunscherfüllende Medizin“ und Krankheitsbegriff: Eine medizintheoretische Analyse, in: Matthias Kettner (Hrsg.), Wunscherfüllende Medizin? (im Druck)

Caplan/Engelhardt/McCartney (1981): Arthur L. Caplan, H. Tristram Engelhardt, James J. McCartney (ed.), Concepts of Health and Disease, Reading 1981

Caplan/McCartney/Sisti (2004): Arthur L. Caplan, James J. McCartney, Dominic A. Sisti (ed.), Health, Disease, and Illness, Washington 2004

Culver/Gert (1982): Charles M. Culver, Bernard Gert, Philosophy in Medicine, New York, Oxford 1982

Doerr/Schipperges (1979): Wilhelm Doerr, Heinrich Schipperges, Was ist Theoretische Pathologie?, Heidelberg 1979

DSM-IV-TR (2003): Diagnostisches und Statistisches Manual Psychischer Störungen - Textrevision, München 2003

Fonagy et al. (2004): Peter Fonagy, György Gergely, Elliot L. Jurist et al., Affektregulierung, Mentalisierung und die Entwicklung des Selbst, Stuttgart 2004

Förstl (2005): Hans Förstl (Hrsg.), Frontalhirn. Funktionen und Erkrankungen, Heidelberg, 2. Aufl., 2005

Förstl (2007): Hans Förstl (Hrsg.), Theory of Mind. Neurobiologie und Psychologie sozialen Verhaltens, Heidelberg 2007

Gert/Culver/Clouser (2006): Bernard Gert, Charles M. Culver, K. Danner Clouser, Bioethics: A Systematic Approach, Oxford 2006

Goffman (1967): Erving Goffman, Stigma, Frankfurt a. M. 1967

Grundmann et al. (2004): Ekkehard Grundmann, Albert Roessner, Ulrich Pfeifer et al. (Hrsg.), Allgemeine Pathologie, München, Jena, 10. Aufl., 2004

Herpertz-Dahlmann et al. (2003): Beate Herpertz-Dahlmann, Franz Resch, Michael Schulte-Markwort et al. (Hrsg.), Entwicklungspsychiatrie. Biopsychologische Grundlagen und die Entwicklung psychischer Störungen, Stuttgart, New York 2003

Hippokrates (1975): (Aus der Schule des) Hippokrates, Die Natur des Menschen, in: Karl E. Rothschuh (Hrsg.), Was ist Krankheit?, Darmstadt 1975, S. 11-18

Hoffmann (2006): Martin Hoffmann, Gibt es eine klare Abgrenzung von Therapie und Enhancement?, Jahrbuch für Wissenschaft und Ethik 11 (2006), S. 201-221 
Hucklenbroich (1998): Peter Hucklenbroich, Die Struktur des medizinischen Wissens. Zur Grundlegung und zum Verhältnis von Medizintheorie und medizinischer Ethik, Zeitschrift für medizinische Ethik 44 (1998), 2, S. $107-125$

Hucklenbroich (2006): Peter Hucklenbroich, Wissenschaftstheoretische Aspekte des medizinischen Krankheitsbegriffs. First Draft, http://egtm.klinikum.uni-muenster.de/mitarbeiter/hucklen/pub.html

Hucklenbroich (2007a): Peter Hucklenbroich, (Hauptartikel:) Krankheit - Begriffsklärung und Grundlagen einer Krankheitstheorie, Erwägen - Wissen - Ethik 18 (2007), 1, S. 77-90

Hucklenbroich (2007 b): Peter Hucklenbroich, (Replik:) Klärungen, Präzisierungen und Richtigstellungen zur Krankheitstheorie, Erwägen - Wissen - Ethik 18 (2007), 1, S. 140-156

Hucklenbroich/Buyx/Suhm (2007): Peter Hucklenbroich, Alena M. Buyx, Christian Suhm (Hrsg.), Wissenschaftstheoretische Aspekte des medizinischen Krankheitsbegriffs, Paderborn 2007 (im Druck)

Humber/Almeder (1997): James M. Humber, Robert F. Almeder (ed.), What is Disease?, Totowa 1997

ICD-10 (2005): Weltgesundheitsorganisation (Hrsg.), Internationale Klassifikation psychischer Störungen. ICD10 Kapitel V (F). Klinisch-diagnostische Leitlinien, Bern 52005

Löffler/Petrides (2003): Georg Löffler, Petro E. Petrides (Hrsg.), Biochemie und Pathobiochemie, Berlin, 7. Aufl., 2003

Lux (2003): Thomas Lux (Hrsg.), Kulturelle Dimensionen der Medizin, Berlin 2003

Markowitsch (2005): Hans I. Markowitsch, Harald Welzer, Das autobiographische Gedächtnis. Hirnorganische Grundlagen und biosoziale Entwicklung, Stuttgart 2005

Mazal (1992): Wolfgang Mazal, Krankheitsbegriff und Risikobegrenzung, Wien 1992

Mazal (2004): Wolfgang Mazal, Krankheit als Rechtsbegriff, in: Nadja Mazouz, Micha H. Werner, Urban Wiesing (Hrsg.), Krankheitsbegriff und Mittelverteilung, Baden-Baden 2004, S. 127-138

Nordenfelt $(1987,1995)$ : Lennart Nordenfelt, On the Nature of Health, Dordrecht 1987, 2. Aufl., 1995

Nordenfelt (2001): Lennart Nordenfelt, Health, Science, and Ordinary Language, Amsterdam 2001

Parsons (1967): Talcott Parsons, Definition von Gesundheit und Krankheit im Lichte der Wertbegriffe und der sozialen Struktur Amerikas, in: Alexander Mitscherlich, Tobias Brocher, Otto von Mering et al. (Hrsg.), Der Kranke in der modernen Gesellschaft, Köln, Berlin 1967, S. 59-87

Petermann (1998): Franz Petermann, Michael Kusch, Kay Niebank, Entwicklungspsychopathologie, Wiesbaden 1998

Petermann (2004): Franz Petermann, Kay Niebank, Herbert Scheithauer, Entwicklungswissenschaft. Entwicklungspsychologie - Genetik - Neuropsychologie, Berlin 2004

Reznek (1987): Lawrie Reznek, The Nature of Disease, London, New York 1987

Riede/Werner/Schaefer (2004): Ursus-Nikolaus Riede, Martin Werner, Hans-Eckart Schaefer (Hrsg.), Allgemeine und spezielle Pathologie, Stuttgart, 5. Aufl., 2004

Rosenthal (2007): Hans A. Rosenthal, Krankheitstheorie und einige praktische Erwägungen, Erwägen Wissen - Ethik 18 (2007), 1, S. $124 \mathrm{f}$.

Rothschuh (1958): Karl E. Rothschuh, Theorie des Organismus. Bios - Psyche - Pathos, München 1958

Rothschuh (1975 a): Karl E. Rothschuh, Der Krankheitsbegriff (was ist Krankheit?), in: Rothschuh (1975 b), S. $397-420$

Rothschuh (1975 b): Karl E. Rothschuh (Hrsg.), Was ist Krankheit?, Darmstadt 1975

Sacks (1995): Oliver Sacks, Eine Anthropologin auf dem Mars, Reinbek 1995

Schipperges (1999): Heinrich Schipperges, Krankheit und Kranksein im Spiegel der Geschichte, Berlin 1999

Schipperges/Seidler/Unschuld (1978): Heinrich Schipperges, Eduard Seidler, Paul U. Unschuld (Hrsg.), Krankheit, Heilkunst, Heilung, München 1978

Thomas (2000): Lothar Thomas (Hrsg.), Labor und Diagnose, Frankfurt a. M., 5. Aufl., 2000

Weber/Stierlin (1989): Guntram Weber, Helm Stierlin, In Liebe entzweit. Ein systemischer Ansatz zum Verständnis und zur Behandlung der Magersuchtsfamilie, Reinbek 1989

Welzer (2006): Harald Welzer, Hans ). Markowitsch (Hrsg.), Warum Menschen sich erinnern können, Stuttgart 2006

Wieland (1986): Wolfgang Wieland, Strukturwandel der Medizin und ärztliche Ethik, Heidelberg 1986 
Wieland (2004): Wolfgang Wieland, Grundlegende Aspekte des Krankheitsbegriffs, in: Nadja Mazouz, Micha H. Werner, Urban Wiesing (Hrsg.), Krankheitsbegriff und Mittelverteilung, Baden-Baden 2004, S. 15-29

Wieland (2007): Wolfgang Wieland, Zur Tragfähigkeit eines wertfreien Krankheitsbegriffs, Erwägen - Wissen Ethik 18 (2007), 1, S. 133-136

Wiesing (1998): Urban Wiesing, Kann die Medizin als praktische Wissenschaft auf eine allgemeine Definition von Krankheit verzichten?, Zeitschrift für Medizinische Ethik 44 (1998), S. 83-97

Wiesing (2004): Urban Wiesing, Kritische Anmerkungen zu einer Krankheitsdefinition anhand objektiver Kriterien, in: Nadja Mazouz, Micha H. Werner, Urban Wiesing (Hrsg.), Krankheitsbegriff und Mittelverteilung, Baden-Baden 2004, S. 47-55

Wiesing (2007): Urban Wiesing, Die Medizin kann auf eine allgemeine Definition von Krankheit verzichten und die Medizintheorie sollte andere Fragen stellen, Erwägen - Wissen - Ethik 18 (2007), 1, S. 136-138 